\title{
Journey to the birth of the Universe
}

This week, astronomers announce the discovery of the most distant galaxy yet seen from Earth. In doing so, they provide fresh clues about the early history of the Universe and seize a hotly contested record. But for some in the field, the competition to bag the record for the most distant object is losing its sparkle.

The new galaxy, dubbed IOK-1, is so far away that the light by which it was detected was emitted only 750 million years after the Universe was born (see page 186). This is just a few hundred million years after the first stars are thought to have switched on.

Other teams have claimed galaxies even farther back in time, although these are awaiting confirmation - or have turned out to be wrong (see 'The galaxy that wasn't'). But the latest detection is not in doubt. "Most people would agree that this is the most convincing, most distant object that has been published," says Richard Ellis of the California Institute of Technology in Pasadena, who also hunts for distant galaxies.

To find the object, Masanori Iye of the National Astronomical Observatory in Tokyo and his colleagues used the 8.2-metre Subaru Telescope in Hawaii to look for the characteristic radiation emitted by hydrogen in hot,

\section{RECORD BREAKERS}

Since the 1950s, astronomers have been pushing the limits of how far away we can see.

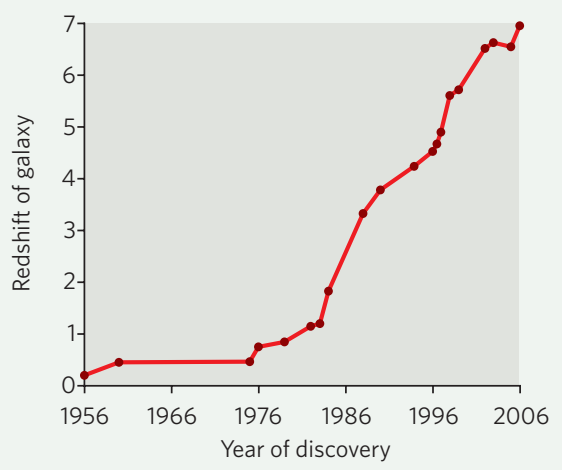

star-forming regions. This emission, known as Lyman- $\alpha$ radiation, has a wavelength that puts it in the ultraviolet part of the spectrum. But light reaching us from distant galaxies has been stretched, or 'redshifted', by the expansion of the Universe, so Iye's team looked for the line at longer wavelengths - the larger the redshift, the older the emitting object. They identified a Lyman-a emitter with a redshift of 6.96, then confirmed it was a galaxy by studying the spectrum of its light in more detail. The identity of

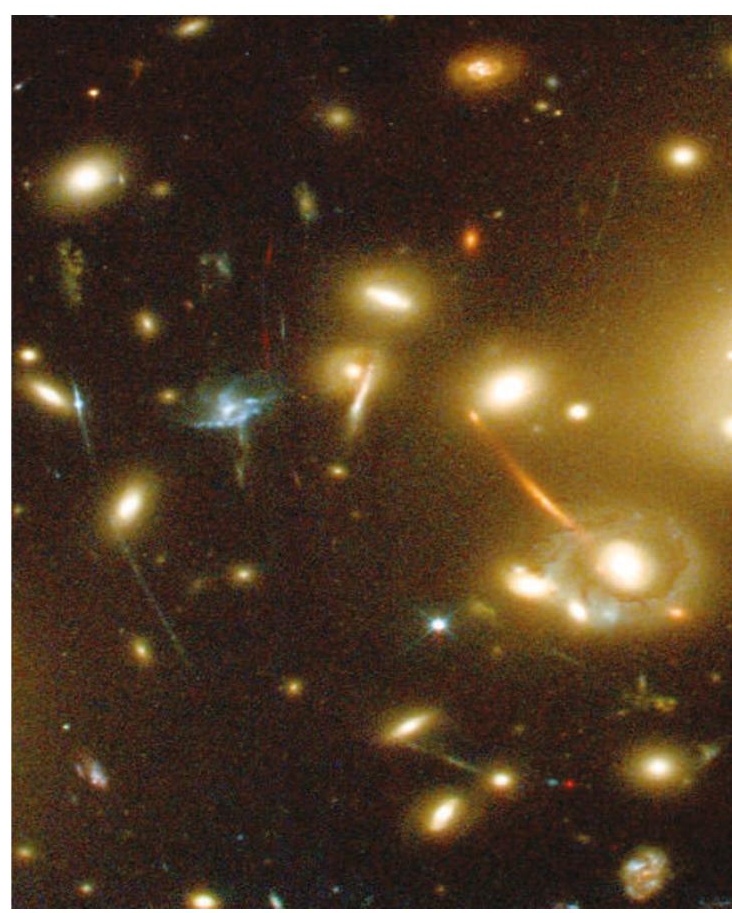

a second emitter remains uncertain.

The redshift of the most distant galaxies known has crept up from a neighbourly 1-2 in the early 1980s to the latest value obtained by Iye (see graph). Each new record has drawn much interest. "This is one of the few areas in observational astronomy where you can get your name in the Guinness Book of Records," notes Richard McMahon of the University of Cambridge, UK, who used to hold the record himself.

\section{Biomedical research gets a fresh twist down on the farm}

\section{ASHBURN, VIRGINIA}

Designers overlooked one small flaw when they drew up plans for Janelia Farm, the new $\$ 500$-million facility for the Howard Hughes Medical Institute in Virginia. Deer, unaware of their new neighbour built into the side of a hill, started leaping over the structure to their deaths. The deer soon wised up. But Janelia's élite group of investigators may hope they aren't taking a comparable leap into the unknown by joining this unusual laboratory.

Janelia Farm, which welcomed its first few investigators in August, trumpets itself as a rare institutional model for innovative biomedical research. The lure, including smart labs, has drawn
16 leading scientists so far, most in neurobiology and image processing, with 44 expected by 2009 . Many surrendered the comforts of tenure for this cutting-edge facility, which pushes scientists to think big and take chances on interdisciplinary collaborations.

But there are risks. Investigators will be reviewed after six years initially, and subsequently every five years. If a panel of outside experts finds the research to be too conventional or progress insufficient, the investigator will be kicked out - albeit with two years of additional funding to soften the blow. "The deal is: you bet your career," says director Gerry Rubin, "and I bet \$10 million."
Those who have come to Janelia are an idiosyncratic bunch. They include Gene Myers, a shotgun gene-sequencing pioneer who gave up tenure at the University of California, Berkeley, to come. Eric Betzig started as a physicist at New Jersey's famed Bell Laboratories before working at his family's machine-tool business for eight years. Sean Eddy, a computational biologist, arrived from a tenured genetics position at Washington University in St Louis, Missouri.

By any metric, Janelia is an impressive lab space. The building is carved into a hill about
60 kilometres west of Washington DC, with each floor in the large, multi-level terrace being at ground level. Labs are walled in with thick glass from floor to ceiling, and a modular design allows them to be "The dealis: easily reconfigured to suit an investigator's needs. you bet your The campus also boasts a career, and I bet gym, 50 apartments and 10 million" a daycare facility. There's also a pub, complete with free coffee to entice people together and a whiteboard for spontaneous brainstorms.

It's all part of a social-engineering project, painstakingly designed to stimulate social interactions and spark informal research discussions. Rubin, a Drosophila 


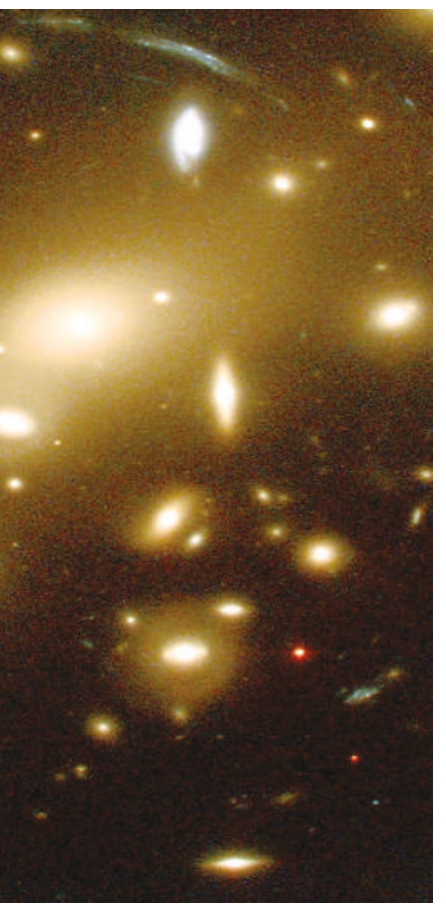

Turning back time: the search for distant galaxies is giving astronomers a clearer picture of how the early Universe behaved.

But some hope to move on from merely claiming records. "I think the field is changing from being simply record-driven, to finding out whether we can reach times where we can see very different properties in the early Universe," says Esther Hu, of the University of Hawaii in Honolulu, who championed early efforts to find Lyman- $\alpha$ emitters.

In this respect, the more interesting aspect of Iye's paper is that the team found fewer galaxies than it expected. This matches a survey

\section{The galaxy that wasn't}

In March 2004, the European Southern Observatory issued a press release with the headline "VLT Smashes the Record of the Farthest Known Galaxy".

A team of astronomers had used the observatory's Very Large Telescope in Chile to study objects whose light was being amplified by the Abell 1835 galactic cluster. The researchers thought they had found a galaxy at redshift 10, or just a few hundred million years after the Universe was born (R. Pelló et al. Astron. Astrophys. 416, L35-L40; 2004). But later analyses of the data cast doubt on this claim, and recently the Spitzer Space Telescope failed to see the object at all.

According to first author
SOUTH KOREANS RACE FOR SPACE

Fast runners qualify for astronaut selection www.nature.com/news by Rychard Bouwens and Garth Illingworth of the University of California, Santa Cruz (see page 189). They analysed data from the Hubble Space Telescope and suggest that the number of detectable galaxies drops off above a redshift of 7 .

This decline might mean that astronomers are getting close to the point when the first galaxies formed. Earlier galaxies may have been smaller, fainter objects that later merged to form brighter beacons. Such information could be fed into simulations of how hydrogen in the early Universe clumped together (see page 151). "What we've done is provide key data that can provide the constraints or inputs to the models," says Illingworth.

That doesn't mean astronomers have given up on going farther back in time. New instruments, including an infrared camera to be installed on the Hubble Space Telescope - if the space shuttle makes another servicing trip - and the James Webb Space Telescope, due to fly in 2013 , should find a flood of more distant objects.

In the meantime, new techniques are pushing back the limits. Ellis, for example, is searching for distant galaxies whose faint light has been amplified by the gravitational fields of closer galactic clusters. He is preparing a paper that claims six candidates around a redshift of 10 - dating from when the Universe was between 400 million and 500 million years old.

Some prefer to investigate closer, brighter galaxies, about which more can be learnt. "Unless you can measure something else in the galaxy, it's not very useful," says Illingworth.

But going for the record does get you attention. A story featuring Ellis's search for the oldest objects made the cover of Time magazine's 4 September issue. Illingworth, an old friend and collaborator, jokes: "It's good for astronomy to have these articles, but I'll kick Richard in the butt sometime for not mentioning the other things we're doing."

Jenny Hogan geneticist who is moving his lab to Janelia from the University of California, Berkeley, based Janelia loosely on the open laboratories of Bell Labs and the Medical Research Council's Laboratory of Molecular Biology in Cambridge, UK.

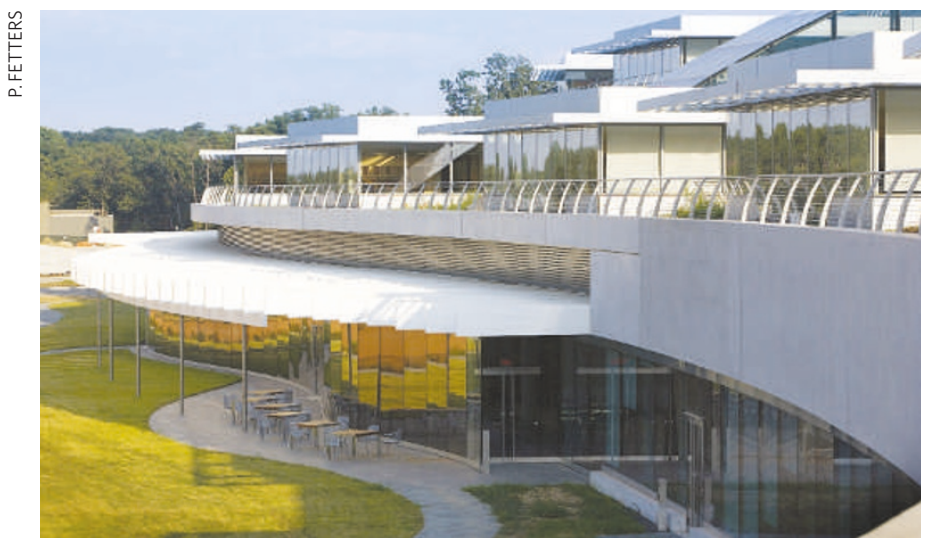

Space to think: the Janelia Farm lab aims to encourage unconventional work.

Groups are no bigger than six members, to encourage interdisciplinary collaboration that relies on field experts rather than graduate students or postdocs. This should also ensure that group leaders are active bench scientists rather than just managers, says Rubin. The glass walls help too, by letting researchers see who's in and what they're doing.

At Bio-X, a similar suite of glasswalled labs at Stanford University in California, few have complained about the lack of privacy, says its operations director Heideh Fattaey. But some have set up whiteboards to block off space. "You can't change human nature," she says. In-house architect Bob McGhee, who helped to design the labs at Janelia, acknowledges that they are not suitable for researchers conducting, for example, ethically sensitive animal studies.

Rubin insists that he doesn't want to replicate other institutes, no matter how successful or prestigious they may be. "My greatest failure would be if this turns into another Salk or Whitehead," he says.

Interdisciplinary labs may be trendy, but placing physicists next to biologists doesn't necessarily spark a revolution. "There's not as much real collaboration as one would think," says Janelia group leader Loren Looger, a protein-engineering researcher with a background in maths and chemistry, who has also worked at Bio-X. But Janelia should prove more successful, he says, as group leaders, already proficient in multiple disciplines, will not have to worry about defending unconventional research to secure tenure - sometimes a problem in academia.

"It may fail," says Looger. Nevertheless, he revels in being part of the Janelia experiment.

Gene Russo 